\title{
Mössbauer Spectroscopy of Fe-Ni-Nb-B Alloy in Weak Magnetic Field
}

\author{
Jozef Sitek, Jarmila Degmová, Katarína Sedlačková, Július Dekan \\ Institute of Nuclear and Physical Engineering, Faculty of Electrical Engineering and Information Technology, \\ Slovak University of Technology, Bratislava, Slovakia \\ Email: jarmila.degmova@stuba.sk
}

Received November 7, 2011; revised December 12, 2011; accepted December 24, 2011

\begin{abstract}
Amorphous and nanocrystalline $\left(\mathrm{Fe}_{1-\mathrm{x}} \mathrm{Ni}_{\mathrm{x}}\right)_{81} \mathrm{Nb}_{7} \mathrm{~B}_{12}(\mathrm{x}=0,0.25,0.5,0.75)$ alloys were measured by Mössbauer spectrometry in the weak external magnetic field of $0.5 \mathrm{~T}$. From structural analyses, ferromagnetic bcc-FeNi and fcc-FeNi and paramagnetic $(\mathrm{Fe}-\mathrm{Ni})_{23} \mathrm{~B}_{6}$ phases were identified in the annealed samples. It was shown that in the external magnetic field the intensities of the $2^{\text {nd }}$ and the $5^{\text {th }}$ lines $\left(\mathrm{A}_{23}\right.$ parameter $)$ are the most sensitive Mössbauer parameters. Rather small changes were observed in the values of internal magnetic field. Our results showed that the amorphous precursor is more sensitive to the influence of external magnetic field than the nanocrystalline alloy. All spectra of amorphous precursor showed the increase of $\mathrm{A}_{23}$ parameter and decrease of internal magnetic field values of about $1 \mathrm{~T}( \pm 0.5 \mathrm{~T})$ under influence of external magnetic field. In the case of nanocrystalline samples the tendency for the values of internal magnetic field is similar but the effect is not so pronounced. The measurements confirmed that even weak external magnetic field affected orientation of the net magnetic moments. Our results indicate that effect of the external magnetic field is stronger in the case of amorphous samples due to their disordered structure.
\end{abstract}

Keywords: Mössbauer Spectroscopy; Nanocrystalline Fe-Ni Alloy; Soft Magnetic Materials

\section{Introduction}

Nanocrystalline alloys rich in Fe prepared from amorphous precursors by crystallization represent an important part of soft magnetic materials. Alloys based on iron and nickel are in the constant focus of interest in physical and material research as well as in industrial applications, as they represent the class of construction materials and show unique magnetic properties. Alloys containing Fe and $\mathrm{Ni}$ together with further additions of specific elements are attractive from mechanical, magnetic and structural point of view [1,2].

Amorphous and more recently also nanocrystalline materials have been studied for applications in magnetic devices requiring magnetically soft materials $[3,4]$. In the last years, alloys with crystallite sizes less than $100 \mathrm{~nm}$ have attracted attention due to some of their magnetic properties such as effective permeability and saturation magnetic flux density $[5,6]$. These properties indicate that these materials may have application in magnetic parts and devices such as inductors, low and high energy frequency transformers, alternating current machines, motors, generators, transducers, sensors and actuators $[7,8]$.

The substitution of small amounts of $\mathrm{Ni}$ for $\mathrm{Fe}$ in $\mathrm{Fe}-$ based magnetic materials generally results in an increase of saturation magnetization [9] and it also leads to a change of structure of nanograins from body-centered cubic (bcc) to face-centered cubic (fcc) and to formation of fcc-type (Fe-Ni)B lattice with a large unit cell $[10,11]$. Recently, in Fe-Ni-Nb-B alloy, the coexistence of bccand fcc-FeNi grains as well as $(\mathrm{Fe}-\mathrm{Ni})_{23} \mathrm{~B}_{6}$ phase have been observed [11]. The analysis of phase evolution upon crystallization indicates that an increase of $\mathrm{Ni}$ content leads to the formation of different ratio of nanocrystalline phases and consequently to a change of magnetic properties.

Up to now, the magnetic parameters derived from Mössbauer spectra of nanocrystalline materials were usually measured as a function of temperature. In most of the cases, the spectra were measured in a strong external magnetic field of several Tesla [12,13]. An influence of weak magnetic field with the strength of less than 1 Tesla on the shape of Mössbauer spectra of nanocrystalline materials were investigated only occasionally [14].

In the present work we studied the influence of the weak external magnetic field on the magnetic microstructure of nanocrystalline $\left(\mathrm{Fe}_{1-\mathrm{x}} \mathrm{Ni}_{\mathrm{x}}\right)_{81} \mathrm{Nb}_{7} \mathrm{~B}_{12}(\mathrm{x}=0$, $0.25,0.5,0.75)$ alloys and their amorphous precursors.

\section{Experimental Details}

Ribbon-shaped specimens of the master alloy were prepared by planar flow casting. The ribbons with nominal 
composition of $\left(\mathrm{Fe}_{1-\mathrm{x}} \mathrm{Ni}_{\mathrm{x}}\right)_{81} \mathrm{Nb}_{7} \mathrm{~B}_{12}(\mathrm{x}=0,0.25,0.5,0.75)$ were about $25 \mu \mathrm{m}$ thick and $10 \mathrm{~mm}$ wide. To achieve nanocrystalline state, the amorphous ribbons were annealed in vacuum at the temperature of $550^{\circ} \mathrm{C}$ for 1 hour. Mössbauer spectra were collected in transmission geometry by a conventional constant-acceleration spectrometer with a ${ }^{57} \mathrm{Co}(\mathrm{Rh})$ source. Mössbauer experiments were performed also in weak external magnetic field up to $0.5 \mathrm{~T}$. The magnetic field was oriented perpendicular with respect to the ribbon plane. All spectra were measured at room temperature and evaluated by the Normos program [15], which allows simultaneous treatment of crystalline and residual amorphous phase by means of individual lines and distribution of hyperfine components. The isomer shift values were quoted relative to the spectrum of $\alpha$-Fe.

\section{Results and Discussion}

Mössbauer spectra of amorphous and nanocrystalline Fe$\mathrm{Ni}-\mathrm{Nb}$-B samples measured with and without application of weak magnetic field are shown in Figures $\mathbf{1}$ and 2. The applied fitting model for amorphous alloy consists of a group of individual subspectra resulting from the distribution of hyperfine parameters. In case of nanocrystalline alloy, a group of narrow lines attributed to the $\mathrm{Fe}$ atoms situated in the bulk of nanocrystalline grains is considered besides the distribution ascribed to amorphous residual matrix. Tables $\mathbf{1}$ and $\mathbf{2}$ summarize behavior of the most sensitive parameters of Mössbauer spectra under influence of weak magnetic field. The results show that the values of the second to third line intensity ratio $\left(A_{23}\right)$ exhibit rather significant changes while the values of the average internal magnetic field (B) vary only slightly.

In the case of amorphous sample, the influence of average internal magnetic field of the amorphous alloy and external weak magnetic field has been considered. For nanocrystalline sample, additionally the internal magnetic field of the crystalline component has to be taken into account, as all these fields interact together and thus influence the orientation of the magnetic domains. We also cannot exclude the presence of interactions among the nanocrystalline grains because these interactions influence the relaxation and magnetization processes relatively strongly. In the alloy with higher content of $\mathrm{Ni}(1: 3)$, the traces of the paramagnetic component are observed. We expect that these paramagnetic areas are bases for later creation of paramagnetic phase of $(\mathrm{Fe}-\mathrm{Ni})_{23} \mathrm{~B}_{6}$ during heat treatment.

The intensity ratio $\mathrm{A}_{23}$ of amorphous phase increases in weak external field, which give us the evidence that the orientation of magnetic domains turns more into direction of the ribbon plane. Higher content of Ni causes more pronounced changes of $\mathrm{A}_{23}$ parameter, which indicates that amorphous precursor with higher concentration of $\mathrm{Ni}$ is more sensitive to weak external magnetic field. The corresponding values of the average internal magnetic field exhibit small changes in the frame of error $( \pm 0.5 \mathrm{~T})$.

The phase analysis of alloy without $\mathrm{Ni}$ or with lower Ni concentration $(3: 1)$ upon nanocrystallization reveals the presence of only bcc-phase embedded in amorphous matrix. With a further increase of $\mathrm{Ni}$ content, the coexistence of the bcc and fcc crystalline phases has been identified [1]. In the sample with high $\mathrm{Ni}$ content $(1: 3)$, the existence of paramagnetic phase of $(\mathrm{Fe}-\mathrm{Ni})_{23} \mathrm{~B}_{6}$ was observed.

The nanocrystalline samples exhibit sensitivity to weak external magnetic field of both amorphous and crystalline components. In the case of the samples without $\mathrm{Ni}$ content, the $2^{\text {nd }}$ to $3^{\text {rd }}$ line intensity ratio of both components increases under influence of external magnetic field. Addition of $\mathrm{Ni}$ causes decrease of $\mathrm{A}_{23}$ parameter in amorphous component and its increase in crystalline part. The changes are quite pronounced for the samples with high content of Ni. These results indicate that the mag-

Table 1. Amorphous Fe-Ni-Nb-B samples.

\begin{tabular}{lccc}
\hline & \multicolumn{3}{c}{ Amorphous } \\
\cline { 2 - 4 } & $\mathrm{B}( \pm 0.5 \mathrm{~T})$ & $\mathrm{A}_{23}( \pm 0.2)$ & $\%( \pm 0.5)$ \\
\hline $1: 0$ & 9.23 & 3.71 & 100 \\
$1: 0^{\mathrm{m}}$ & 9.34 & 3.88 & 100 \\
$3: 1$ & 20.25 & 2.09 & 100 \\
$3: 1^{\mathrm{m}}$ & 19.96 & 3.34 & 100 \\
$1: 1$ & 20.04 & 1.82 & 100 \\
$1: 1^{\mathrm{m}}$ & 19.81 & 3.27 & 100 \\
$1: 3^{*}$ & 15.52 & 0.93 & 98 \\
$1: 3^{\mathrm{m} *}$ & 15.89 & 2.37 & 94 \\
\hline
\end{tabular}

${ }_{\mathrm{m}} \mathrm{MS}$ measurement with applied magnetic field; ${ }^{*}$ Mössbauer spectrum contains also quadrupole doublets.

Table 2. Nanocrystalline Fe-Ni-Nb-B samples.

\begin{tabular}{ccccccc}
\hline & \multicolumn{3}{c}{ Amorphous } & \multicolumn{3}{c}{ Crystalline } \\
\cline { 2 - 7 } Fe-Ni & $\mathrm{B}$ & $\mathrm{A}_{23}$ & $\%$ & $\mathrm{~B}$ & $\mathrm{~A}_{23}$ & $\%$ \\
$\pm 0.5 \mathrm{~T}$ & \pm 0.2 & $\pm 0.5)$ & $\pm 0.5 \mathrm{~T}$ & \pm 0.2 & \pm 0.5 \\
\hline $1: 0$ & 9.91 & 1.12 & 84 & 32.96 & 2.23 & 16 \\
$1: 0^{\mathrm{m}}$ & 10.00 & 1.82 & 85 & 32.97 & 3.17 & 15 \\
$3: 1$ & 19.35 & 3.64 & 89 & 33.67 & 3.08 & 11 \\
$3: 1^{\mathrm{m}}$ & 18.71 & 3.31 & 86 & 33.37 & 3.30 & 14 \\
$1: 1$ & 13.81 & 1.26 & 70 & 28.69 & 4 & 30 \\
$1: 1^{\mathrm{m}}$ & 13.59 & 1.04 & 71 & 28.70 & 3.4 & 29 \\
$1: 3^{*}$ & 22.74 & 1.66 & 47 & 29.80 & 4 & 20 \\
$1: 3^{\mathrm{m}^{*}}$ & 9.79 & 1.03 & 46 & 28.90 & 4 & 28 \\
\hline
\end{tabular}

${ }^{\mathrm{m}} \mathrm{MS}$ measurement with applied magnetic field; * Mössbauer spectrum contains also quadrupole doublets. 


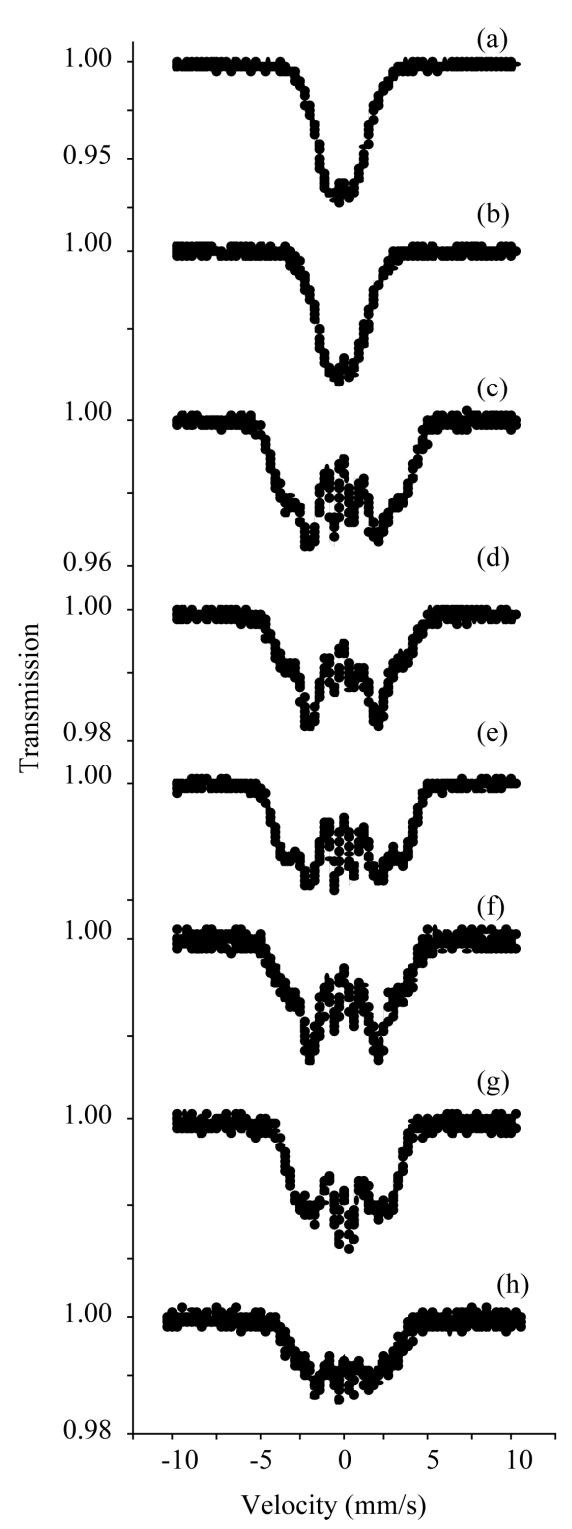

Figure 1. Spectra of amorphous Fe-Ni-Nb-B alloys, where (a) and (b) corresponds to 1:0 ratio of $\mathrm{Fe}: \mathrm{Ni}$; (c) and (d) to 3:1; (e) and (f) to 1:1; (g) and (h) to 1:3. The spectra (b), (d), (f) and (h) were taken in the weak external magnetic field.

netic domains in amorphous part of the sample are more sensitive to the external magnetic field than those in the crystalline region. We suppose that the original magnetic interactions (without influence of external field) in amorphous part are weaker, than in the crystalline one, whatcould explain such a behavior. The corresponding value of average internal magnetic field exhibits small changes in amorphous component as well as in crystalline component where these changes are in the frame of error.

To understand better the magnetic behavior of nanocrystalline materials in weak magnetic field we could apply the theory of magnetic dynamic for non-purely crystalline materials. This theory is based on the general

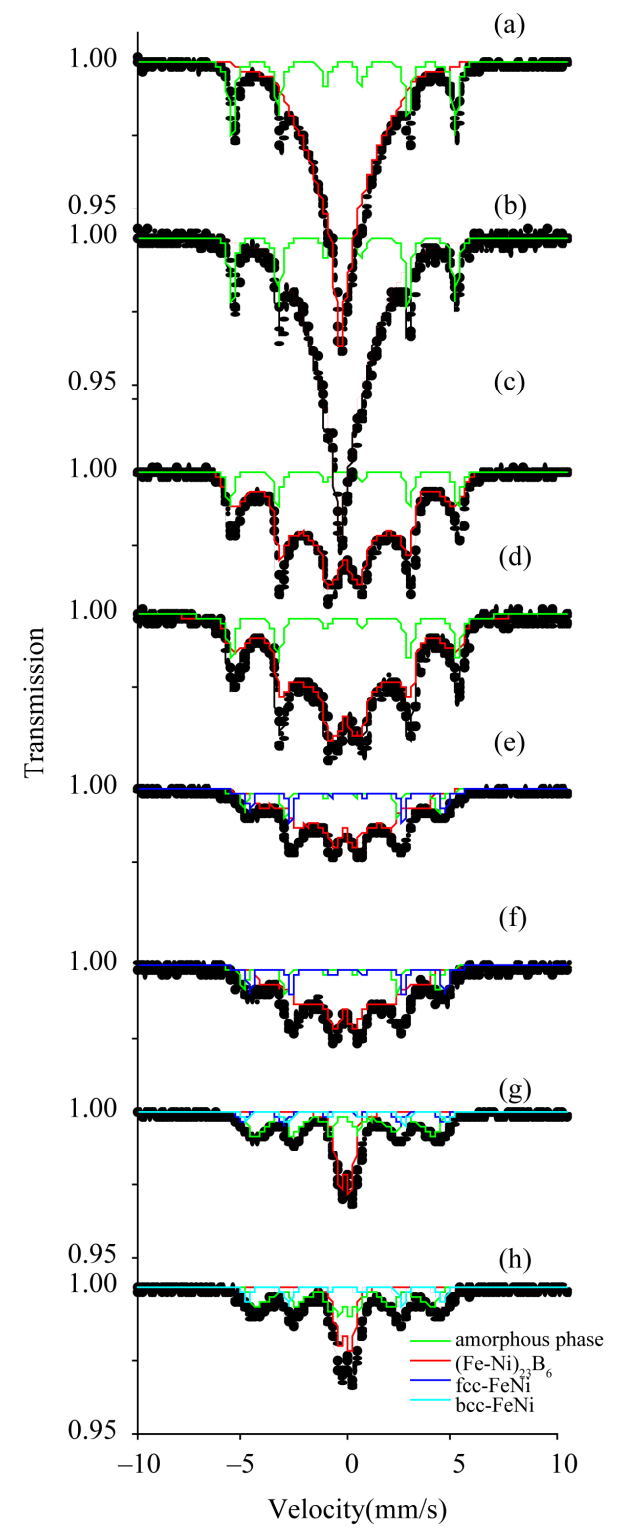

Figure 2. Spectra of nanocrystalline Fe-Ni-Nb-B alloys, where (a) and (b) corresponds to 1:0 ratio of $\mathrm{Fe}: \mathrm{Ni}$; (c) and (d) to $3: 1$; (e) and (f) to $1: 1$; (g) and (h) to $1: 3$. The spectra (b), (d), (f) and (h) were taken in the weak external magnetic field.

equations of stochastic relaxation which is reduced to a three-level relaxation model [16]. This model allows one not only to analyze (in the first approximation) the experimental Mössbauer spectra of alloys in a magnetic field, but also to make qualitative conclusions on specific shapes of the absorption spectra taking into consideration physical mechanisms of formation of the magnetic hyperfine structure within the magnetic dynamics inherent to such materials. A number of qualitative effects observed in experimental Mössbauer spectra can be selfconsistently explained within the model in terms of the mean-field interparticle interaction. In particular, this 
model predicts the appearance of ${ }^{57} \mathrm{Fe}$ magnetic sextets with a small hyperfine splitting slightly dependent on the particle size and temperature in a weak magnetic field and at high temperature, which look like effective "doublets" of lines often observed in experimental spectra. The speeding up and slowing down relaxation in a magnetic field (or in the presence of the meanfield interaction) can also play role in Mössbauer spectra.

In this context it is necessary to emphasize that the three level model [16] was created for nanoparticles i.e. in our case one should take into account beside nanocrystalline grains also influence of amorphous component.

The influence of the weak magnetic field on the alloys, in general, is significantly dependent on the angle between direction of magnetization and external magnetic field. According to the fact that our external magnetic field is constant, we supposed that the changes in the shape of spectra are caused by variation in the value of effective energy barrier i.e. in anisotropy constant. This fact could be also reflected in parameters of Mössbauer spectra, mostly in $\mathrm{A}_{23}$ parameter.

\section{Conclusion}

Our results indicate that in amorphous and nanocrystalline $\left(\mathrm{Fe}_{1-\mathrm{x}} \mathrm{Ni}_{\mathrm{x}}\right)_{81} \mathrm{Nb}_{7} \mathrm{~B}_{12}(\mathrm{x}=0,0.25,0.5,0.75)$ alloys weak external magnetic field has influence on the orientation of the net magnetic moment which is reflected in parameters of Mössbauer spectra. Sensitivity is dependent on the content of Ni. Amorphous component is more sensitive than the crystalline one. This behavior could be explained by weaker magnetic interactions in amorphous state.

\section{Acknowledgements}

"VEGA $1 / 0606 / 09,1 / 0366 / 12$ and 1/0119/12". The idea of the co-author is to have it in this way: "VEGA $1 / 0286 / 12$, $1 / 0366 / 12$ and $1 / 0119 / 12$ ". It is just to change the identification of the first grant as well because it is just old one...

\section{REFERENCES}

[1] G. Vlasak, P. Svec, M. Kuzminski, A. Slawska-Waniewska, B. Butvinova, P. Butvin and J. Hosko, "Evolution of Physical Properties of Amorphous Fe-Ni-Nb-B Alloys with Different Ni/Fe Ratio upon Thermal Treatment," Journal of Alloys and Compounds, Vol. 509, Suppl. 1, 2011, pp. S64-S68.

[2] M. Arshed, M. Siddique, M. Anwar-ul-Islam, A. Ashfaq, A. Shamim and N. M. Butt, "Isochronal Crystallization of Metglass $\mathrm{Fe}_{83} \mathrm{~B}_{17}$ Using Mössbauer Effect and Resistivity Measurements," Solid State Communications, Vol. 98, No. 5, 1996, pp. 427-430. doi:10.1016/0038-1098(96)00025-7

[3] M. E. McHenry, M. A. Willard and D. E. Laughlin, "Amorphous and Nano-Crystalline Materials for Applications as Soft Magnets," Progress in Materials Science, Vol. 44,
No. 4, 1999, pp. 291-433. doi:10.1016/S0079-6425(99)00002-X

[4] M. L. Sui, L. H. Qian and K. Y. He, "Nanocrystallization of Ni-Fe-Nb-Si-B Amorphous Alloys," Materials Science and Engineering: A, Vol. 286, No. 1, 2000, pp. 201-204. doi:10.1016/S0921-5093(00)00714-0

[5] M. Kopcewicz, A. Grabias and D. L. Williamson, "Magnetism and Nano-Structure of $\mathrm{Fe}_{93-\mathrm{x}-\mathrm{y}} \mathrm{Zr}_{7} \mathrm{~B}_{\mathrm{x}} \mathrm{Cu}_{\mathrm{y}}$ Alloys," Journal of Applied Physics, Vol. 82, No. 4, 1997, pp. 1747-1759.

[6] J. S. Garitaonandia, P. Gorria, L. F. Barquín and J. M. Barandiarán, "Low-Temperature Magnetic Properties of Fe Nanograins in an Amorphous Fe-Zr-B Matrix," Physical Review B, Vol. 61, No. 9, 2000, pp. 6150-6155.

[7] J. Bonastre, L. Escoda, A. González, J. Saurina and J. J. Suňol, "Influence of Ni Content on Fe-Nb-B Alloy Formation," Journal of Thermal Analysis and Calorimetry, Vol. 88, No. 1, 2007, pp. 83-86.

doi:10.1007/s10973-006-8092-2

[8] G. Vlasak, P. Svec, M. Kuzminski, A. Slawska-Waniewska, B. Butvinova and P. Butvin, "Magnetic Measurements of Fe-Ni-Nb-B and Fe-Co-Mo-Cu-B in the Vicinity of the Curie Temperature," Journal of Magnetism and Magnetic Materials, Vol. 322, No. 14, 2010, pp. 20472050. doi:10.1016/j.jmmm.2010.01.032

[9] Y. I. Jang, J. Kim and D. H. Shin, "Microstructures and Magnetic Properties of Amorphous Fe-Si-B-Ni Alloy Ribbons," Materials Science and Engineering: B, Vol. 78, No. 2-3, 2000, pp. 113-118.

[10] M. Kopcewicz, B. Idzikowski and J. Kalinowska, "Mössbauer Study of the Magnetism and Structure of Amorphous and Nanocrystalline $\mathrm{Fe}_{81-\mathrm{x}} \mathrm{Ni}_{\mathrm{x}} \mathrm{Zr}_{7} \mathrm{~B}_{12}(\mathrm{x}=10$ - 40) Alloys," Journal of Applied Physics, Vol. 94, No. 1, 2003, pp. 638-650. doi:10.1063/1.1578701

[11] P. Svec, M. Miglierini, J. Dekan, J. Turcanová, G. Vlasák, I. Skorvánek and D. Janickovic, "Influence of Structure Evolution on Magnetic Properties of Fe-Ni-Nb-B System," IEEE Transactions on Magnetics, Vol. 46, No. 2, 2010, pp. 412-415. doi:10.1109/TMAG.2009.2034332

[12] X. Y. Sun, L. Zhen, C. Y. Xu, L. X. Lv, W. Z. Shao and X. D. Sun, "Mössbauer Spectrometry Study of Early Stage Spinodal Decomposition in Fe-Cr-Co Alloy under High Magnetic Field," Materials Letters, Vol. 63, No. 1, 2009, pp. 64-65. doi:10.1016/j.matlet.2008.09.007

[13] M. Reissner, E. Bauer, W. Steiner and Peter Rogl, "High Field Mössbauer and Magnetic Investigations of $\operatorname{Pr}_{0.73}$ $\mathrm{Fe}_{4} \mathrm{Sb}_{12}$," Journal of Magnetism and Magnetic Materials, Vol. 272-276, 2004, pp. 813-815. doi:10.1016/j.jmmm.2003.12.1259

[14] M. A. Polikarpov, V. M. Cherepanov, M. A. Chuev, S. Y. Shishkov and S. S. Yakimov, "Super-Ferromagnetism of Magnetite Nanoparticles in a Weak Magnetic Field," In: H. Muller, M. Reissner, W. Steiner and G. Wiesinger, Eds., ICAME Proceedings, Institute of Solid State Physics, Vienna, 19-24 July 2009, p. 297.

[15] R. A. Brand, "NORMOS Program," 1997, Unpublished.

[16] M. A. Chuev, "Mossbauer Spectra of Single-Domain Particles in a Weak Magnetic Field," Journal of Physics: Condensed Matter, Vol. 20, No. 50, 2008, p. 10. 